International Journal of Advanced Mathematical Sciences, 5(1) (2017) 27-35
International Journal of Advanced Mathematical Sciences
SPC
$\begin{gathered}\text { Website: } w \text { ww.sciencepubco.com/index.php/IJAMS } \\ \text { doi: } 10.14419 / \text { ijams.v5il.6879 } \\ \text { Research paper }\end{gathered}$

\title{
Derivations of Second Type of Algebra of Second Class Filiform Leibniz Algebras of Dimension Derivation $(n+2)$
}

\author{
AL-Nashri AL-hossain Ahmad ${ }^{1^{*}}$ \\ ${ }^{1}$ Umm AL Qura University (K.S.A), AL Qunfudha University college, Department of Mathematics \\ ${ }^{*}$ Corresponding author E-mail:hossa-1427@hotmail.com
}

\begin{abstract}
Keywords: Filiform Leibniz algebra, Leibniz algebra, gradation, natural gradation, derivation.

\section{Introduction}

Many mathematicians have actively studied the theory of Lie algebra for years. J.-L. Loday was the first Mathematician who introduces Leibniz algebra In (1993). Leibniz algebra is a generation of Lie algebra, and it is very common that any associative algebra motivates Lie algebra and Leibniz of algebras $[x, y]=x y-y x$. Actually, it was J.-L. Loday who suggested this new notion of algebra, which was one of the second class filiform Leibniz algebras. This paper discusses the low dimension of algebras. It aims to get the basis of the space $\operatorname{Der}\left(L_{n}(b)\right)$. By discussing the table of algebra from dimension 5 to 16 ,this paper will also try to discover the basis of the derivation of this algebra and the link between the algebra and its derivations.
\end{abstract}

In this article, the derivations of second type of algebra from the second class filiform Leibniz algebras of dimension derivation $(n+2)$ is discussed. From the description of the derivations.It is found that the basis of the space $\operatorname{Der}\left(L_{n}(b)\right)$ of the algebra.

\section{Preliminary Results}

Some definitions and essential results, needed in this paper are prrsented.

Definition 2.1. (see[6]) An algebra L over a field $K$ is called a Leibniz algebra if its bilinear operation [.,.] satisfies the following Leibniz identity:

$$
[x,[y, z]]=[[x, y], z]-[[x, z], y], \text { for any } x, y, z \in L .
$$

in other word, all algebras are assumed to be over the fields of complex numbers $\mathbb{C}$. Now let $L$ be a Leibniz algebra. We put: $L^{1}=L, L^{k+1}=\left[L^{k}, L\right], k \geqslant 1$.

Definition 2.2. (see[8]) A Leibniz algebra $L$ is said to be nilpotent if there exists $s \in N$ such that $L^{1} \supset L^{2} \supset \cdots \supset L^{s}=0$.

Definition 2.3. (see[1]) A Leibniz algebra $L$ is said to be filiform if $\operatorname{dim} L^{i}=n-i$, where $n=\operatorname{dim} L$ and $2 \leqslant i \leqslant n$.

Definition 2.4. (see[7])

A $k$-linear transformation d of an algebra $L$ is called a derivation of $L$ if

$$
d([x, y])=[d(x), y]+[x, d(y)] \text { for all } x, y \in L .
$$

The set of all derivations of an algebra $L$ is denoted by $\operatorname{Der}(L)$. We also, denote by Leibn the set of all $(n+1)$-dimensional filiform Leibniz algebras. We now look at the following theorem from (see[3])which splits the set of fixed dimension filiform Liebniz algebras into three disjoint subsets.However we just take the result of this theorem regarding only $S L e i b_{n+1}$.

Theorem 2.1. Any $(n+1)$-dimensional complex filiform Leibniz algebra $L$ admits a basis $e_{0}, e_{1}, \cdots, e_{n}$ called adapted, such that the table of multiplication of $L$ has the following forms, where non defined products are zero:

$$
\text { SLei }_{n+1}=\left\{\begin{array}{l}
{\left[e_{0}, e_{0}\right]=e_{2},} \\
{\left[e_{i}, e_{0}\right]=e_{i+1}, \quad i \in \llbracket 2 ; n-1 \rrbracket,} \\
{\left[e_{0}, e_{1}\right]=\sum_{i=3}^{n} \beta_{i} e_{i}} \\
{\left[e_{1}, e_{1}\right]=\gamma e_{n-1}} \\
{\left[e_{j}, e_{1}\right]=\sum_{i=3}^{n} \beta_{i} e_{j+i-1}, j \in \llbracket 2 ; n-2 \rrbracket .}
\end{array}\right.
$$

for $\beta_{3}, \beta_{4}, \cdots, \beta_{n}, \beta \in C$.

Lemma 2.1. [7] Let $d \in \operatorname{Der}\left(L_{n}\right)$. In this case $d=\sum_{i=0}^{n-1} d_{i}$ where $d_{k} \in \operatorname{End}\left(L_{n}\right)$ and $d_{k}\left(L_{i}\right) \subseteq L_{i+k}$ for $i \in \llbracket 1 ; n \rrbracket$.

Proof. Consider the natural filtration $S_{i}$ of the algebra $L_{n}$. It can be easely seen from [ (see[7], Corollary 1)] that this filtration coincides with the natural filtration of the algebra $L_{n}$. There for, $S_{k}=\bigoplus_{i \geq k} L_{n}$ and $d\left(S_{i}\right) \subseteq S_{i}$ by [(see[7],Corollary 3)] So,

$d \in F_{0} Z^{1}\left(L_{n}, L_{n}\right)$ implies $d_{k} \in \operatorname{End}\left(L_{n}\right)$ and $d_{k}\left(L_{i}\right) \subseteq L_{i+k}$.

This proves the lemma. 
pr

\section{Algebra $L_{n}(b)$ of the Second Class Filiform Leibniz Algebras}

We denote by $L_{n}(b)$ an algebra of second class filiform Leibniz algebras, and it is defined by

$$
L_{n}(b)=\left\{\begin{array}{l}
{\left[e_{0}, e_{0}\right]=e_{2},} \\
{\left[e_{i}, e_{0}\right]=e_{i+1}, \quad i \in \llbracket 2, n-1 \rrbracket,} \\
{\left[e_{1}, e_{1}\right]=e_{n} .}
\end{array}\right.
$$

Where $\llbracket \mathrm{n} ; \mathrm{m} \rrbracket$ denotes all integers between $n$ and $m$. Tables (1), (2) and (3) (see Appendix) presents the equation (dim Der), number equation of derivation and dim Der of Algebra in Low dimension: Remarks:

In this work, we add some points to give more information about our study.

- A basis of $\operatorname{Der}\left(L_{n}(b)\right)$ can be found and this will be the main purpose of the next pages.

- Based on, $\operatorname{dim} \operatorname{Der}\left(L_{n}(b)\right)=n+2, n \geqslant 4$ the dimension of Der can be computed.

- According to Tables 1,2 and 3, we suggest that the number of equations follows the rule:

Number of equation $=\frac{(n+1)(n+2)}{2}+n$.

- In the next we will present some Lemma and proposition that are the main task of our paper.

Definition 3.1. Let $n \geqslant 4, X=\sum_{i=0}^{n} \lambda_{i} e_{i}$ we denote by

$$
t_{1}(X)=\sum_{i=0}^{n} \lambda_{i} t_{1}\left(e_{i}\right)
$$

where,

$\left\{\begin{array}{l}t_{1}\left(e_{0}\right)=e_{0}, \\ t_{1}\left(e_{1}\right)=\frac{n}{2} e_{1}, \\ t_{1}\left(e_{i}\right)=i e_{i}, i \in \llbracket 2 ; n-1 \rrbracket .\end{array}\right.$

and we define $d_{0}$ such that

$d_{0}\left(e_{i}\right)=\left\{\begin{array}{l}\alpha_{0} e_{0}, \quad i=0, \\ \alpha_{1} \frac{n}{2} e_{1} \quad i=1, \\ \alpha_{i} i e_{i}, \quad i \in \llbracket 2, n \rrbracket .\end{array}\right.$

Lemma 3.1. We have $d_{0}=\alpha_{0} t_{1}$.

Proof. Consider $d_{0} \in \operatorname{Der}\left(L_{n}(b)\right)$ which is defined by where

$\alpha_{0}, \alpha_{1}$, and $\alpha_{i} i \in \llbracket 2, n \rrbracket$ are parameters.

Consider the family of derivations

$d_{0}\left(\left[e_{i}, e_{j}\right]\right)=\left[d_{0}\left(e_{i}\right), e_{j}\right]+\left[e_{i}, d_{0}\left(e_{j}\right)\right], \quad i, j=0,1,2, \cdots, n-1$.

We now look at the problem case by case. In each case, we repeatedly use algebra $L_{n}(b)$ and (1).

- First Case: if $(i, j)=(0,0)$

then

$d_{0}\left(\left[e_{0}, e_{0}\right]\right)=\left[d_{0}\left(e_{0}\right), e_{0}\right]+\left[e_{0}, d_{0}\left(e_{0}\right)\right]$

so,

$d_{0}\left(e_{2}\right)=\left[\alpha_{0} e_{0}, e_{0}\right]+\left[e_{0}, \alpha_{0} e_{0}\right]$

which implies

$2 \alpha_{2} e_{2}=\alpha_{0} e_{2}+\alpha_{0} e_{2}$

thus

$$
\alpha_{2}=\alpha_{0}
$$

- Second Case: if $(i, j) \in(\llbracket 2 ; n \rrbracket, 0)$

then

$d_{0}\left(\left[\sum_{i=2}^{n} e_{i}, e_{0}\right]\right)=\left[d_{0}\left(\sum_{i=2}^{n} e_{i}\right), e_{0}\right]+\left[\sum_{i=2}^{n} e_{i}, d_{0}\left(e_{0}\right)\right]$

so that,

$d_{0}\left(\sum_{i=2}^{n-1} e_{i+1}\right)=\left[\sum_{i=2}^{n} \alpha_{i} e_{i}, e_{0}\right]+\left[\sum_{i=2}^{n} e_{i}, \alpha_{0} e_{0}\right]$

which implies

$\sum_{i=2}^{n-1} \alpha_{i+1}(i+1) e_{i+1}=\sum_{i=2}^{n-1} i \alpha_{i} e_{i+1}+\sum_{i=2}^{n-1} \alpha_{0} e_{i+1}$

$\sum_{i=2}^{n-1} \alpha_{i+1}(i+1) e_{i+1}=\sum_{i=2}^{n-1}\left(i \alpha_{i}+\alpha_{0}\right) e_{i+1}$

and so,

$$
(i+1) \alpha_{i+1}=i \alpha_{i}+\alpha_{0}, \quad i \in \llbracket 2 ; n-1 \rrbracket .
$$

If $\mathrm{i}=2$ in 3 then $3 \alpha_{3}=2 \alpha_{2}+\alpha_{0}$ By (2) we obtain

$$
\alpha_{3}=\alpha_{2}
$$

If $\mathrm{i}=3$ in 3 then $4 \alpha_{4}=3 \alpha_{3}+\alpha_{0}$ By ( 2 and 4 ) we obtain

$$
\alpha_{4}=\alpha_{3}
$$

Similarly,

If $i=n-1$ in 3 then

$$
\text { (n) } \alpha_{n}=(n-1) \alpha_{n-1}+\alpha_{0}
$$

but $\alpha_{0}=\alpha_{n-1}$

Thus,

$$
\alpha_{n}=\alpha_{n-1}
$$

From (2),(4),(5)and (6) we obtain

$$
\alpha_{0}=\alpha_{2}=\alpha_{3}=\alpha_{4}=\cdots=\alpha_{n} .
$$

Thus

$$
\begin{aligned}
d_{0}\left(\sum_{i=0}^{n} \lambda_{i} e_{i}\right) & =d_{0}\left(\lambda_{0} e_{0}\right)+d_{0}\left(\lambda_{1} e_{1}\right)+d_{0}\left(\lambda_{2} e_{2}\right)+\sum_{i=3}^{n-1} d_{0}\left(\lambda_{i} e_{i}\right) \\
& =\lambda_{0}\left(\alpha_{0} e_{0}\right)+\lambda_{1}\left(\alpha_{1} \frac{n}{2} e_{1}\right)+2 \lambda_{2}\left(\alpha_{2} e_{2}\right)+\sum_{i=3}^{n-1} i \lambda_{i}\left(\alpha_{i} e_{i}\right) \\
b y(1) & =\lambda_{0}\left(\alpha_{0} e_{0}\right)+\lambda_{1}\left(\alpha_{1} \frac{n}{2} e_{1}\right)+\sum_{i=2}^{n-1} i \lambda_{i}\left(\alpha_{i} e_{i}\right) \\
& =\alpha_{0}\left[\lambda_{0} e_{0}+\lambda_{1} \frac{n}{2} e_{1}+\sum_{i=2}^{n-1} i \lambda_{i} e_{i}\right] \quad b y(7) \\
& =\alpha_{0} t_{1} .
\end{aligned}
$$

From this we have

$t_{1}\left(e_{0}\right)=e_{0}, \quad t_{1}\left(e_{1}\right)=\frac{n}{2} e_{1}$ and $t_{1}\left(e_{i}\right)=i e_{i}, \quad i \in \llbracket 2 ; n-1 \rrbracket$.

from this we have our result

Lemma 3.2. Consider $d_{k} \in \operatorname{Der}\left(L_{n}(b)\right), \quad k \in \llbracket 1, n-2 \rrbracket, n \geqslant 3$ such that $d_{k}$ is defined by

$d_{k}\left(e_{i}\right)=\left\{\begin{array}{l}\lambda_{0} e_{k}, \quad i=0, k \in \llbracket 1, n \rrbracket, \\ \lambda_{i} e_{k+i-1}, \quad i \in \llbracket 2, n-k \rrbracket .\end{array}\right.$

where $\lambda_{0}$ and $\lambda_{i}, \quad i \in \llbracket 1, n-k \rrbracket$, are scalars.

Then $d_{k}\left(e_{0}\right)=e_{k} \quad, \quad d_{k}\left(e_{i}\right)=e_{i+k-1}, \quad i \in \llbracket 2, n-k \rrbracket$, and $k \in \llbracket 1, n-2 \rrbracket$. 
Proof. Consider the family of derivations

$d_{k}\left(\left[e_{i}, e_{j}\right]\right)=\left[d_{k}\left(e_{i}\right), e_{j}\right]+\left[e_{i}, d_{k}\left(e_{j}\right)\right]$.

Similarly, we calculate case by case.In which we repeatedly $L_{n}(a)$ and (9).

Case $1:$ if $i=0, j=0$ then

$\sum_{k=1}^{n-2} d_{k}\left(\left[e_{0}, e_{0}\right]\right)=\left[\sum_{k=1}^{n-2} d_{k}\left(e_{0}\right), e_{0}\right]+\left[e_{0}, \sum_{k=1}^{n-2} d_{k}\left(e_{0}\right)\right]$.

Then

$\sum_{k=1}^{n-2} d_{k}\left(e_{2}\right)=\left[\sum_{k=1}^{n-2} \lambda_{0} e_{k}, e_{0}\right]+\left[e_{0}, \sum_{k=1}^{n-2} \lambda_{0} e_{k}\right]$

which implies

$\sum_{k=1}^{n-2} \lambda_{2} e_{k+1}=\sum_{k=1}^{n-2} \lambda_{0} e_{k+1}$

to obtain

$$
\lambda_{2}=\lambda_{0}
$$

Case $2: \operatorname{if}(i, j)=(\llbracket 1, n-1 \rrbracket, 0)$ and $k \in \llbracket 1, n-2 \rrbracket$, then

$\sum_{k=1}^{n-2} \sum_{i=1}^{n-1} d_{k}\left(\left[e_{i}, e_{0}\right]\right)=\left[\sum_{k=1}^{n-2} \sum_{i=1}^{n-1} d_{k}\left(e_{i}\right), e_{0}\right]+\left[\sum_{i=1}^{n-1} e_{i}, \sum_{k=2}^{n-1} d_{k}\left(e_{0}\right)\right]$

$\sum_{k=1}^{n-2} \sum_{i=2}^{n-2} d_{k}\left(e_{i+1}\right)=\left[\sum_{k=1}^{n-2} \sum_{i=1}^{n-1} \lambda_{i} e_{k+i-1}, e_{0}\right]+\left[\sum_{i=1}^{n-1} e_{i}, \sum_{k=1}^{n-2} d_{k}\left(e_{0}\right)\right]$

$\sum_{k=1}^{n-2} \sum_{i=1}^{n-2} \lambda_{i+1}\left(e_{i+k}\right)=\left[\sum_{k=1}^{n-2} \sum_{i=1}^{n-1} \lambda_{i} e_{k+i-1}, e_{0}\right]+\left[\sum_{i=1}^{n-1} e_{i}, \sum_{k=1}^{n-2} \lambda_{0}\left(e_{k}\right)\right]$

$\sum_{k=1}^{n-2} \sum_{i=1}^{n-2} \lambda_{i+1} e_{i+k}=\sum_{k=1}^{n-2} \sum_{i=1}^{n-2} \lambda_{i} e_{k+i}$

Thus,

$\lambda_{i+1}=\lambda_{i}$

From (11)

if $i=2$ then $\lambda_{3}=\lambda_{2}$

also,

if $i=3$ then $\lambda_{4}=\lambda_{3}$

similarly,

if $i=n-1$ then $\lambda_{n}=\lambda_{n-1}$

From (10) and (11) this implies

$$
\lambda_{0}=\lambda_{2}=\lambda_{3}=\ldots=\lambda_{n-1}=\lambda_{n}
$$

and hence,

$$
\begin{aligned}
d_{k}\left(\sum_{i=0}^{n} \alpha_{i} e_{i}\right) & =d_{k}\left(\alpha_{0} e_{0}\right)+d_{k}\left(\alpha_{1} e_{1}\right)+\sum_{i=2}^{n} d_{k}\left(\alpha_{i} e_{i}\right) \\
& =\alpha_{0}\left(\lambda_{0} e_{k}\right)+\sum_{i=2}^{n} \lambda_{i}\left(\alpha_{i} e_{k+i-1}\right) \quad b y(9) \\
& =\alpha_{0}\left(\lambda_{0} e_{k}+\sum_{i=2}^{n-k} \lambda_{i} e_{k+i}\right) \quad \text { by }(7) .
\end{aligned}
$$

Thus,

$$
d_{k}\left(e_{0}\right)=e_{k} \text { and } d_{k}\left(e_{i}\right)=e_{k+i}, i \in \llbracket 2, n-k \rrbracket .
$$

Lemma 3.3.

$$
\text { Let } X=\sum_{i=0}^{n} \lambda_{i} e_{i}
$$

Then, $t_{2}(X)=\sum_{i=0}^{n} \lambda_{i} t_{2}\left(e_{i}\right)$, where $\delta_{0}^{i} e_{n}$ and $\delta_{0}^{i}$ is the Kronecker symbol.

Proof. Consider $d_{n-1} \in \operatorname{Der}\left(L_{n}(a)\right)$ where $d_{n-1}$ is defined by

$d_{n-1}\left(e_{i}\right)=\left\{\begin{array}{cc}\pi_{0} e_{n}, & i=0 \\ 0 ; & i \neq 0\end{array}\right.$

for scalar $\pi_{0}$.

Consider the family of derivations

$d_{n-1}\left(\left[e_{i}, e_{j}\right]\right)=\left[d_{n-1}\left(e_{i}\right), e_{j}\right]+\left[e_{i}, d_{n-1}\left(e_{j}\right)\right]$

Case $1:$ if $i=0, j=0$ then

$d_{n-1}\left(\left[e_{0}, e_{0}\right]\right)=\left[d_{n-1}\left(e_{0}\right), e_{0}\right]+\left[e_{0}, d_{n-1}\left(e_{0}\right)\right]$

by $L_{n}(b)$ and (13), thus

$d_{n-1}\left(e_{2}\right)=\left[\pi_{0} e_{n}, e_{0}\right]+\left[e_{0}, \pi_{0} e_{n}\right]$.

If $\pi_{0} \neq 0$ then

$0=0+0$

Hence

$$
\begin{aligned}
d_{n-1}\left(\sum_{i=0}^{n} \lambda_{i} e_{i}\right) & =d_{n-1}\left(\lambda_{0} e_{0}\right)+d_{n-1}\left(\lambda_{1} e_{1}\right)+\sum_{i=2}^{n} d_{n-1}\left(\lambda_{i} e_{i}\right) \\
& =\lambda_{0}\left(\pi_{0} e_{n}\right) \text { by }(14) \\
& =\pi_{0}\left(\lambda_{0} e_{n}\right) \\
& =\pi_{0} t_{2}
\end{aligned}
$$

and we obtain

$$
t_{2}\left(e_{0}\right)=e_{n}
$$

And here we obtain the result.

Thus we have our result. 
Lemma 3.4. The mappings $t_{1}, t_{2}$ and $d_{k}$ for $1 \leq k \leq(n-2)$ are linearly independent.

Proof. Consider that

$\alpha_{1} t_{1}\left(e_{i}\right)+\alpha_{2} t_{2}\left(e_{i}\right)+\sum_{k=1}^{n-2} \beta_{k} d_{k}\left(e_{i}\right)=0$

where $e_{i} \in L_{n}(a), i=0,1,2,3, \ldots, n-1$.

We will show that $\alpha_{1}=\alpha_{2}=\beta_{1}=\beta_{2}=\ldots=\beta_{k}=0$ for $1 \leq k \leq n-2$.

$$
\begin{aligned}
& \sum_{i=0}^{n-1}\left[\alpha_{1} t_{1}\left(e_{i}\right)+\alpha_{2} t_{2}\left(e_{i}\right)+\sum_{k=1}^{n-2} \beta_{k} d_{k}\left(e_{i}\right)\right] \\
& =\sum_{i=0}^{n-1}\left[\alpha_{1} t_{1}\left(e_{i}\right)+\alpha_{2} t_{2}\left(e_{i}\right)+\beta_{1} d_{1}\left(e_{i}\right)+\beta_{2} d_{2}\left(e_{i}\right)+\ldots\right. \\
& \left.+\beta_{n-3} d_{n-3}\left(e_{i}\right)+\beta_{n-2} d_{n-2}\left(e_{i}\right)\right] \\
& =\alpha_{1} t_{1}\left(e_{0}\right)+\alpha_{2} t_{2}\left(e_{0}\right)+\beta_{1} d_{1}\left(e_{0}\right)+\beta_{2} d_{2}\left(e_{0}\right)+\ldots \\
& +\beta_{n-3} d_{n-3}\left(e_{0}\right)+\beta_{n-2} d_{n-2}\left(e_{0}\right) \\
& +\left[\alpha_{1} t_{1}\left(e_{1}\right)+\alpha_{2} t_{2}\left(e_{1}\right)+\beta_{1} d_{1}\left(e_{1}\right)+\beta_{2} d_{2}\left(e_{1}\right)+\ldots\right. \\
& \left.+\beta_{n-3} d_{n-3}\left(e_{1}\right)+\beta_{n-2} d_{n-2}\left(e_{1}\right)\right] \\
& +\left[\alpha_{1} t_{1}\left(e_{2}\right)+\alpha_{2} t_{2}\left(e_{2}\right)+\beta_{1} d_{1}\left(e_{2}\right)+\beta_{2} d_{2}\left(e_{2}\right)+\ldots\right. \\
& \left.+\beta_{n-3} d_{n-3}\left(e_{2}\right)+\beta_{n-2} d_{n-2}\left(e_{2}\right)\right] \\
& \left.+\beta_{n-3} d_{n-3}\left(e_{n-1}\right)+\beta_{n-2} d_{n-2}\left(e_{n-1}\right)\right] \\
& + \\
& + \\
& +
\end{aligned}
$$

This implies

$\left(\alpha_{1}\left(e_{0}+(2 n-5) e_{1}\right)+\left(\alpha_{1}(n-1)+2 \beta_{1}\right) e_{2}+\left(n \alpha_{1}+2 \beta_{2}+\beta_{1}\right) e_{3}\right.$

$+\left((n+1) \alpha_{1}+2 \beta_{3}+\beta_{2}+\beta_{1}\right) e_{4}$

$+\left((n+2) \alpha_{1}+2 \beta_{4}+\beta_{3}+\beta_{2}+\beta_{1}\right) e_{5}+\ldots$

$+\left(2(n-2) \alpha_{1}+2 \beta_{n-2}+\beta_{n-1}+\ldots+\beta_{3}+\beta_{2}+\beta_{1}\right) e_{n-1}$

$+\left((n-1)\left(\alpha_{1}+3\right)+\alpha_{2}+2 \beta_{n-2}+\beta_{n-1}+\ldots+\beta_{3}+\beta_{2}+\beta_{1}\right) e_{n}$

$=0$

Here we have these following results:

1) $\alpha_{1} e_{1}=0$ which implies $\alpha_{1}=0$.

2) $\left(\alpha_{1}(n-1)+2 \beta_{1}\right) e_{2}=0$ which implies $\alpha_{1}(n-1)+2 \beta_{1}=0$ but since $\alpha_{1}=0$ then $\beta_{1}=0$.

3) $\left(n \alpha_{1}+2 \beta_{2}+\beta_{1}\right) e_{3}=0$ which implies $n \alpha_{1}+2 \beta_{2}+\beta_{1}=0$ but since $\alpha_{1}=\beta_{1}=0$ then $\beta_{2}=0$.

4) $\left((n-1) \alpha_{1}+2 \beta_{3}+\beta_{2}+\beta_{1}\right) e_{4}=0$

which implies $(n-1) \alpha_{1}+2 \beta_{3}+\beta_{2}+\beta_{1}=0$

but since $\alpha_{1}=\beta_{1}=\beta_{2}=0$ then $\beta_{3}=0$.

5) $\left((n-2) \alpha_{1}+2 \beta_{4}+\beta_{2}+\beta_{3}+\beta_{1}\right) e_{5}=0$

which implies $\left((n-2) \alpha_{1}+2 \beta_{4}+\beta_{2}+\beta_{3}+\beta_{1}\right)=0$

but since $\alpha_{1}=\beta_{1}=\beta_{2}=\beta_{3}=0$ then $\beta_{4}=0$.

Similarly,

6) $\left(2(n-2) \alpha_{1}+2 \beta_{n-2}+\beta_{n-1}+\beta_{n}+\ldots+\beta_{1}\right) e_{n-1}=0$

which implies $\left(2(n-2) \alpha_{1}+2 \beta_{n-2}+\beta_{n-1}+\beta_{n}+\ldots+\beta_{1}\right)=0$

but since $\alpha_{1}=2 \beta_{n-2}=\beta_{n-1}=\ldots=\beta_{1}=0$ then $\beta_{n-2}=0$.

7) $\left((n-2)\left(\alpha_{1}+3\right)+\alpha_{2}+2 \beta_{n}+\beta_{n-1}+\beta_{n}+\ldots+\beta_{1}\right) e_{n}=0$ which implies

$\left((n-2)\left(\alpha_{1}+3\right)+\alpha_{2}+2 \beta_{n-1}+\beta_{n-1}+\beta_{n}+\ldots+\beta_{1}\right)=0$

but since $\alpha_{1}=\beta_{n-1}=\beta_{n-2}=\beta_{n-3}=\ldots=\beta_{2}=\beta_{1}=0$,

then

$\alpha_{2}=0$.

From above we will obtain

$\alpha_{1}=\alpha_{2}=\beta_{1}=\beta_{2}=\beta_{3}=\beta_{4}=\beta_{5}=\ldots=\beta_{n-1}=0$.

Those prove the mappings are linearly independent.

Lemma 3.5. The linear mappings $d_{0}, d_{k}, d_{n-1} \in \operatorname{Der}\left(L_{n}(b)\right), 1 \leq$ $k \leq n-2$ defined by (1), (9) and (14) are linearly composition. 


\section{Proof. Let}

$$
x=\eta_{0} e_{0}+\eta_{1} e_{1}+\eta_{2} e_{2}+\eta_{3} e_{3}+\eta_{4} e_{4}+\ldots+\eta_{n-1} e_{n-1} .
$$

First we observe that by (1), (9) and (14),

$d_{k}(x)=\left\{\begin{array}{l}2 \eta_{0}\left(\alpha_{1} e_{1}\right)+\eta_{1}\left(\alpha_{0}(n-1)\right) e_{1}+\sum_{2}^{n} \alpha_{i}\left(\eta_{i} i e_{i}\right) \\ +\sum_{k=1}^{n} \eta_{0} \lambda_{0} e_{k}+\sum_{k=2}^{n-1} \sum_{i=2}^{n-1} \lambda_{i} \eta_{i} e_{k+i-1}+\eta_{0} \pi_{0} e_{n} .\end{array}\right.$

Hence by using (7) and (12),

$d(x)=\alpha_{0}\left[\eta_{0}\left(e_{1}\right)+\eta_{1}(n-1) e_{1}+\sum_{2}^{n} \eta_{i} i e_{i}\right]$

$+\lambda_{0}\left[\sum_{k=1}^{n} \eta_{0} e_{k}+\sum_{k=2}^{n-1} \sum_{i=2}^{n-1} \eta_{i} e_{k+i-1}\right]+\pi_{0} \eta_{0} e_{n}$.

Thus

$$
d=\alpha_{0} t_{1}+\pi_{0} t_{2}+\lambda_{0} d_{k} \text { for } 1 \leq k \leq n-2 .
$$

This prove linear composition of the mappings.

Lemma 3.6. The mappings $t_{1}, t_{2}, d_{k}$ for $1 \leq k \leq n-2$ are derivations.

Proof. Consider that

$$
x=\alpha_{0} e_{0}+\alpha_{1} e_{1}+\alpha_{2} e_{2}+\alpha_{3} e_{3}+\ldots+\alpha_{n-1} e_{n-1}
$$

and

$$
y=\beta_{0} e_{0}+\beta_{1} e_{1}+\beta_{2} e_{2}+\beta_{3} e_{3}+\ldots+\beta_{n-1} e_{n-1}
$$

Then

$$
x . y=\beta_{0}\left(\alpha_{0} . e_{2}+\alpha_{2} e_{3}+\alpha_{3} e_{4}+\ldots+\left(\alpha_{n-1}+\alpha_{1}\right) e_{n}\right.
$$

and so,

$t_{1}(x . y)=\beta_{0}\left(2 \alpha_{0} e_{2}+3 \alpha_{2} e_{3}+4 \alpha_{3} e_{4}+\ldots+n\left(\alpha_{n-1}+\alpha_{1}\right) e_{n}\right.$

Thus,

$t_{1}(x)=\alpha_{0} e_{0}+\alpha_{1}(n-1) e_{1}+2 \alpha_{2} e_{2}+3 \alpha_{3} e_{3}+\ldots+\alpha_{n-1}(n-1) e_{n-1}$

Hence

$t_{1}(x) . y=\beta_{0}\left[\alpha_{0} e_{2}+2 \alpha_{2} e_{3}+3 \alpha_{3} e_{4}+\ldots+(n-1)\left(\alpha_{n-1}+\alpha_{1}\right) e_{n}\right]$ and thus,

$t_{1}(y)=\beta_{0} e_{0}+\beta_{1}(n-1) e_{1}+2 \beta_{2} e_{2}+3 \beta_{3} e_{3}+\ldots+\beta_{n-1}(n-1) e_{n-1}$

Therefore,

$$
x . t_{1}(y)=\beta_{0}\left[\alpha_{0} e_{2}+\alpha_{2} e_{3}+\ldots+\left(\alpha_{n-1}+\alpha_{1}\right) e_{n}\right]
$$

By adding (20) to (21) we will obtain (19). This implies $t_{1}$ is a derivation.

We now show that $t_{2}$ is also a derivation .

$$
\begin{aligned}
& t_{2}(x)=\alpha_{0} e_{n} \\
& t_{2}(y)=\beta_{0} e_{n}
\end{aligned}
$$

From easy calculation we have $t_{2}(x) \cdot y=0, x \cdot t_{2}(y)=0$ and $t_{2}(x \cdot y)=$ 0

and thus $t_{2}$ is a derivation.

Now, since

$d_{k}(x)=\sum_{k=1}^{n-1}\left[\alpha_{0} d_{k}\left(e_{0}\right)+\alpha_{1} d_{k}\left(e_{1}\right)+\alpha_{2} d_{k}\left(e_{2}\right)+\ldots+\alpha_{n-1} d_{k}\left(e_{n-1}\right)\right]$

thus,

$$
\sum_{k=1}^{n-1} d_{k}(x)=\sum_{k=1}^{n-1}\left[\alpha_{0} e_{k}+\sum_{i=2}^{n-k} \alpha_{i} e_{k+i-1}\right]
$$

then,

$$
\begin{gathered}
{\left[d_{k}(x) . y\right]=\beta_{0}\left[\sum _ { k = 1 } ^ { n - 1 } \left(e_{k+1}+\sum_{k=1}^{n-1}\left(\alpha_{2} e_{k+2}+\alpha_{3} e_{k+3}\right.\right.\right.} \\
\left.\left.\left.+\ldots+\alpha_{n-k} e_{n-k}\right)\right)\right] .
\end{gathered}
$$

In addition,

$$
\sum_{k=1}^{n-1} d_{k}(y)=\sum_{k=1}^{n-1}\left[\alpha_{0} e_{k}+\sum_{i=2}^{n-k} \alpha_{i} e_{k+i-1}\right]
$$

and we have

$$
\left[x, \sum_{k=1}^{n-1} d_{k}(y)\right]=0
$$

Also,

$\sum_{k=1}^{n-1} d_{k}(x \cdot y)=\sum_{k=1}^{n-1} \beta_{0}\left(\alpha_{0} e_{k+1}+\alpha_{2} e_{k+2}+\alpha_{3} e_{k+3}+\ldots+\alpha_{n-k} e_{n-1}\right.$

By adding (22) to (23) we will get (24),thus $d_{k}$ is a derivation. This completes the proof of the proposition.

The following is our main result. we note that $t_{1}, t_{2}$, and $d_{k}$ for $1 \leq k \leq n-2$ are defined in Lemma 3.1 to Lemma 3.3.

Proposition 3.1. Let $L_{n}(b)$ be $e_{0} e_{0}=e_{2}, e_{i} e_{0}=e_{i+1}, 2 \leq i \leq n-1$ and $e_{1} e_{0}=e_{n}$. Then $t_{1}, t_{2}$ and $d_{k}$ for $1 \leq k \leq n-2$ form a basis of the space $\operatorname{Der}\left(L_{n}(b)\right)$.

Proof. The proof follows from Lemma 3.4 to Lemma 3.6

\section{Conclusion}

1. This algebra $L_{n}(b)$, is nilpotent, but it is not characteristically nilpotent.

2. This algebra $L_{n}(b)$ work with basis derivations from five dimension and above.

3. We can find number derivations of this algebra on any dimension by this rule:

$$
\operatorname{dim} \operatorname{Der}\left(L_{n}(b)\right)=n+2
$$

4. We can determine number of equations from the result of derivations by this rule number equations $=\frac{(n+1)(n+2)}{2}+n$

\section{Appendix}


Table 1: Dimension derivation of Algebra from 5 to 9.

\begin{tabular}{|c|c|c|c|}
\hline dimension & equation(dim Der) & dim Der & No.of equations \\
\hline 5 & $\begin{array}{c}d_{1}\left(e_{0}\right)=e_{0}, \quad d_{1}\left(e_{1}\right)=2 e_{1}, \\
d_{1}\left(e_{i}\right)=i e_{i}, 2 \leqslant i \leqslant 4 \\
d_{2}\left(e_{i}\right)=0,0 \leqslant i \leqslant 4 \\
d_{3}\left(e_{0}\right)=e_{2}, \quad d_{3}\left(e_{1}\right)=0, d_{3}\left(e_{i}\right)=e_{i+1}, \quad 2 \leqslant i \leqslant 3 \\
d_{4}\left(e_{0}\right)=e_{3}, d_{4}\left(e_{1}\right)=0, d_{4}\left(e_{2}\right)=e_{4}, \\
d_{5}\left(e_{0}\right)=e_{4}, d_{6}\left(e_{1}\right)=e_{4} .\end{array}$ & 6 & 19 \\
\hline 6 & $\begin{array}{c}d_{1}\left(e_{0}\right)=e_{0}, d_{1}\left(e_{1}\right)=\frac{5}{2} e_{1}, \\
d_{1}\left(e_{i}\right)=i e_{i}, 2 \leqslant i \leqslant 5 \\
d_{2}\left(e_{i}\right)=0,0 \leqslant i \leqslant 5 \\
d_{3}\left(e_{0}\right)=e_{2}, \quad d_{3}\left(e_{1}\right)=0, \quad d_{3}\left(e_{i}\right)=e_{i+1}, 2 \leqslant i \leqslant 4 \\
d_{4}\left(e_{0}\right)=e_{3}, \quad d_{4}\left(e_{1}\right)=0, \quad d_{4}\left(e_{i}\right)=e_{i+2}, 2 \leqslant i \leqslant 3 \\
d_{5}\left(e_{0}\right)=e_{4}, d_{5}\left(e_{1}\right)=0, d_{5}\left(e_{2}\right)=e_{5}, \\
d_{6}\left(e_{0}\right)=e_{5}, d_{7}\left(e_{1}\right)=e_{5} .\end{array}$ & 7 & 26 \\
\hline 7 & $\begin{array}{c}d_{1}\left(e_{0}\right)=e_{0}, d_{1}\left(e_{1}\right)=3 e_{1}, \\
d_{1}\left(e_{i}\right)=i e_{i}, 2 \leqslant i \leqslant 6 \\
d_{2}\left(e_{i}\right)=0,0 \leqslant i \leqslant 6 \\
d_{3}\left(e_{0}\right)=e_{2}, d_{3}\left(e_{1}\right)=0, \quad d_{3}\left(e_{i}\right)=e_{i+1}, 2 \leqslant i \leqslant 5 \\
d_{4}\left(e_{0}\right)=e_{3}, d_{4}\left(e_{1}\right)=0, \quad d_{4}\left(e_{i}\right)=e_{i+2}, 2 \leqslant i \leqslant 4 \\
d_{5}\left(e_{0}\right)=e_{4}, d_{5}\left(e_{1}\right)=0, d_{5}\left(e_{i}\right)=e_{i+3}, 2 \leqslant i \leqslant 3 \\
d_{6}\left(e_{0}\right)=e_{5}, d_{6}\left(e_{1}\right)=0, d_{6}\left(e_{2}\right)=e_{6} \\
d_{7}\left(e_{1}\right)=e_{6} d_{7}\left(e_{1}\right)=e_{6} .\end{array}$ & 8 & 34 \\
\hline 8 & $\begin{array}{c}d_{1}\left(e_{0}\right)=e_{0}, d_{1}\left(e_{1}\right)=\frac{7}{2} e_{1}, \\
d_{1}\left(e_{i}\right)=i e_{i}, 2 \leqslant i \leqslant 7 \\
d_{2}\left(e_{i}\right)=0,0 \leqslant i \leqslant 7 \\
d_{3}\left(e_{0}\right)=e_{2}, \quad d_{3}\left(e_{1}\right)=0, d_{3}\left(e_{i}\right)=e_{i+1}, 2 \leqslant i \leqslant 6 \\
d_{4}\left(e_{0}\right)=e_{3}, d_{4}\left(e_{1}\right)=0, d_{4}\left(e_{i}\right)=e_{i+2}, 2 \leqslant i \leqslant 5 \\
d_{5}\left(e_{0}\right)=e_{4}, d_{5}\left(e_{1}\right)=0, d_{5}\left(e_{i}\right)=e_{i+3}, \quad 2 \leqslant i \leqslant 4 \\
d_{6}\left(e_{0}\right)=e_{5}, d_{6}\left(e_{1}\right)=0, d_{6}\left(e_{2}\right)=e_{i+4}, \quad 2 \leqslant i \leqslant 3 \\
d_{7}\left(e_{0}\right)=e_{6} d_{7}\left(e_{0}\right)=0 d_{7}\left(e_{2}\right)=e_{7} \\
d_{8}\left(e_{0}\right)=e_{7} d_{9}\left(e_{1}\right)=e_{7} .\end{array}$ & 9 & 43 \\
\hline 9 & $\begin{array}{c}d_{1}\left(e_{0}\right)=e_{0}, d_{1}\left(e_{1}\right)=4 e_{1}, \\
d_{1}\left(e_{i}\right)=i e_{i}, 2 \leqslant i \leqslant 8 \\
d_{2}\left(e_{i}\right)=0,0 \leqslant i \leqslant 8, \\
d_{3}\left(e_{0}\right)=e_{2}, d_{3}\left(e_{1}\right)=0, d_{3}\left(e_{i}\right)=e_{i+1}, 2 \leqslant i \leqslant 7 \\
d_{4}\left(e_{0}\right)=e_{3}, d_{4}\left(e_{1}\right)=0, d_{4}\left(e_{i}\right)=e_{i+2}, 2 \leqslant i \leqslant 6 \\
d_{5}\left(e_{0}\right)=e_{4}, d_{5}\left(e_{1}\right)=0, d_{5}\left(e_{i}\right)=e_{i+3}, \quad 2 \leqslant i \leqslant 5 \\
d_{6}\left(e_{0}\right)=e_{5}, d_{6}\left(e_{1}\right)=0, d_{6}\left(e_{2}\right)=e_{i+4}, \quad 2 \leqslant i \leqslant 4 \\
d_{7}\left(e_{0}\right)=e_{6}, d_{7}\left(e_{1}\right)=0 d_{7}\left(e_{i}\right)=e_{i+5}, \quad 2 \leqslant i \leqslant 3 \\
d_{8}\left(e_{0}\right)=e_{7}, d_{8}\left(e_{1}\right)=0, d_{8}\left(e_{2}\right)=e_{8}, \\
d_{9}\left(e_{0}\right)=e_{8}, d_{10}\left(e_{0}\right)=e_{8} .\end{array}$ & 10 & 53 \\
\hline
\end{tabular}


Table 2: Dimension derivation of Algebra from 10 to 13.

\begin{tabular}{|c|c|c|c|}
\hline Dimension & Equation(dim Der) & dim Der & No.of equations \\
\hline 10 & $\begin{array}{c}d_{1}\left(e_{0}\right)=e_{0}, d_{1}\left(e_{1}\right)=4 e_{1}, \\
d_{1}\left(e_{i}\right)=i e_{i}, 2 \leqslant i \leqslant 9 \\
d_{2}\left(e_{i}\right)=0, \quad 0 \leqslant i \leqslant 9 \\
d_{3}\left(e_{0}\right)=e_{2}, d_{3}\left(e_{1}\right)=0, \quad d_{3}\left(e_{i}\right)=e_{i+1}, 2 \leqslant i \leqslant 8 \\
d_{4}\left(e_{0}\right)=e_{3}, d_{4}\left(e_{1}\right)=0, \quad d_{4}\left(e_{i}\right)=e_{i+2}, 2 \leqslant i \leqslant 7 \\
d_{5}\left(e_{0}\right)=e_{4}, d_{5}\left(e_{1}\right)=0, d_{5}\left(e_{i}\right)=e_{i+3}, \quad 2 \leqslant i \leqslant 6 \\
d_{6}\left(e_{0}\right)=e_{5}, d_{6}\left(e_{1}\right)=0, d_{6}\left(e_{2}\right)=e_{i+4}, \quad 2 \leqslant i \leqslant 5 \\
d_{7}\left(e_{0}\right)=e_{6}, \quad d_{7}\left(e_{1}\right)=0 \quad d_{7}\left(e_{i}\right)=e_{i+5}, \quad 2 \leqslant i \leqslant 4 \\
d_{8}\left(e_{0}\right)=e_{7}, d_{8}\left(e_{1}\right)=0, d_{8}\left(e_{2}\right)=e_{i+6}, \quad 2 \leqslant i \leqslant 3 \\
d_{9}\left(e_{0}\right)=e_{8}, d_{9}\left(e_{1}\right)=0, d_{9}\left(e_{2}\right)=e_{9}, \\
d_{10}\left(e_{0}\right)=e_{9} d_{11}\left(e_{1}\right)=e_{9} .\end{array}$ & 10 & 64 \\
\hline 11 & $\begin{array}{c}d_{1}\left(e_{0}\right)=e_{0}, d_{1}\left(e_{1}\right)=5 e_{1}, \\
d_{1}\left(e_{i}\right)=i e_{i}, 2 \leqslant i \leqslant 10 \\
d_{2}\left(e_{i}\right)=0, \quad 0 \leqslant i \leqslant 10, \quad d_{3}\left(e_{0}\right)=e_{2} \\
d_{3}\left(e_{1}\right)=0, d_{3}\left(e_{i}\right)=e_{i+1}, 2 \leqslant i \leqslant 9 \\
d_{4}\left(e_{0}\right)=e_{3}, \quad d_{4}\left(e_{1}\right)=0, \quad d_{4}\left(e_{i}\right)=e_{i+2}, 2 \leqslant i \leqslant 8 \\
d_{5}\left(e_{0}\right)=e_{4}, \quad d_{5}\left(e_{1}\right)=0, \quad d_{5}\left(e_{i}\right)=e_{i+3}, \quad 2 \leqslant i \leqslant 7 \\
d_{6}\left(e_{0}\right)=e_{5}, \quad d_{6}\left(e_{1}\right)=0, \quad d_{6}\left(e_{2}\right)=e_{i+4}, \quad 2 \leqslant i \leqslant 6 \\
d_{7}\left(e_{0}\right)=e_{6}, \quad d_{7}\left(e_{1}\right)=0 \quad d_{7}\left(e_{i}\right)=e_{i+5}, \quad 2 \leqslant i \leqslant 5 \\
d_{8}\left(e_{0}\right)=e_{7}, \quad d_{8}\left(e_{1}\right)=0, \quad d_{8}\left(e_{2}\right)=e_{i+6}, \quad 2 \leqslant i \leqslant 4 \\
d_{9}\left(e_{0}\right)=e_{8}, \quad d_{9}\left(e_{1}\right)=0, \quad d_{9}\left(e_{2}\right)=e_{i+7}, \quad 2 \leqslant i \leqslant 3 \\
d_{10}\left(e_{0}\right)=e_{9} d_{10}\left(e_{1}\right)=0 d_{10}\left(e_{2}\right)=e_{10} \\
d_{11}\left(e_{0}\right)=e_{10}, d_{12}\left(e_{1}\right)=e_{10}\end{array}$ & 12 & 76 \\
\hline 12 & $\begin{array}{c}d_{1}\left(e_{0}\right)=e_{0}, \quad d_{1}\left(e_{1}\right)=\frac{11}{2} e_{1}, \\
d_{1}\left(e_{i}\right)=i e_{i}, 2 \leqslant i \leqslant 11 \\
d_{2}\left(e_{i}\right)=0, \quad 0 \leqslant i \leqslant 11, d_{3}\left(e_{0}\right)=e_{2}, \\
d_{3}\left(e_{1}\right)=0, \quad d_{3}\left(e_{i}\right)=e_{i+1}, 2 \leqslant i \leqslant 10 \\
d_{4}\left(e_{0}\right)=e_{3}, d_{4}\left(e_{1}\right)=0, \quad d_{4}\left(e_{i}\right)=e_{i+2}, \quad 2 \leqslant i \leqslant 9 \\
d_{5}\left(e_{0}\right)=e_{4}, d_{5}\left(e_{1}\right)=0, \quad d_{5}\left(e_{i}\right)=e_{i+3}, \quad 2 \leqslant i \leqslant 8 \\
d_{6}\left(e_{0}\right)=e_{5}, \quad d_{6}\left(e_{1}\right)=0, \quad d_{6}\left(e_{2}\right)=e_{i+4}, \quad 2 \leqslant i \leqslant 7 \\
d_{7}\left(e_{0}\right)=e_{6}, \quad d_{7}\left(e_{1}\right)=0 \quad d_{7}\left(e_{i}\right)=e_{i+5}, \quad 2 \leqslant i \leqslant 6 \\
d_{8}\left(e_{0}\right)=e_{7}, \quad d_{8}\left(e_{1}\right)=0, \quad d_{8}\left(e_{2}\right)=e_{i+6}, \quad 2 \leqslant i \leqslant 5 \\
d_{9}\left(e_{0}\right)=e_{8}, \quad d_{9}\left(e_{1}\right)=0, \quad d_{9}\left(e_{2}\right)=e_{i+7}, \quad 2 \leqslant i \leqslant 4 \\
d_{10}\left(e_{0}\right)=e_{9}, \quad d_{10}\left(e_{1}\right)=0, \quad d_{10}\left(e_{2}\right)=e_{i+8}, \quad 2 \leqslant i \leqslant 3 \\
d_{11}\left(e_{0}\right)=e_{10}, d_{11}\left(e_{1}\right)=0, d_{11}\left(e_{2}\right)=e_{11}, \\
d_{12}\left(e_{0}\right)=e_{11}, d_{13}\left(e_{1}\right)=e_{11} .\end{array}$ & 13 & 89 \\
\hline 13 & $\begin{array}{c}d_{1}\left(e_{0}\right)=e_{0}, d_{1}\left(e_{1}\right)=6 e_{1}, \\
d_{1}\left(e_{i}\right)=i e_{i}, 2 \leqslant i \leqslant 12 \\
d_{2}\left(e_{i}\right)=0,0 \leqslant i \leqslant 12, d_{3}\left(e_{0}\right)=e_{2}, \\
d_{3}\left(e_{1}\right)=0, d_{3}\left(e_{i}\right)=e_{i+1}, 2 \leqslant i \leqslant 11 \\
d_{4}\left(e_{0}\right)=e_{3}, d_{4}\left(e_{1}\right)=0, d_{4}\left(e_{i}\right)=e_{i+2}, 2 \leqslant i \leqslant 10 \\
d_{5}\left(e_{0}\right)=e_{4}, d_{5}\left(e_{1}\right)=0, d_{5}\left(e_{i}\right)=e_{i+3}, 2 \leqslant i \leqslant 9 \\
d_{6}\left(e_{0}\right)=e_{5}, d_{6}\left(e_{1}\right)=0, d_{6}\left(e_{2}\right)=e_{i+4}, 2 \leqslant i \leqslant 8 \\
d_{7}\left(e_{0}\right)=e_{6}, d_{7}\left(e_{1}\right)=0 d_{7}\left(e_{i}\right)=e_{i+5}, 2 \leqslant i \leqslant 7 \\
d_{8}\left(e_{0}\right)=e_{7}, d_{8}\left(e_{1}\right)=0, d_{8}\left(e_{2}\right)=e_{i+6}, 2 \leqslant i \leqslant 6 \\
d_{9}\left(e_{0}\right)=e_{8}, d_{9}\left(e_{1}\right)=0, d_{9}\left(e_{2}\right)=e_{i+7}, 2 \leqslant i \leqslant 5 \\
d_{10}\left(e_{0}\right)=e_{9}, d_{10}\left(e_{1}\right)=0, d_{10}\left(e_{2}\right)=e_{i+8}, 2 \leqslant i \leqslant 4 \\
d_{11}\left(e_{0}\right)=e_{10}, d_{11}\left(e_{1}\right)=0, d_{11}\left(e_{i}\right)=e_{i+9}, 2 \leqslant i \leqslant 3 \\
d_{12}\left(e_{0}\right)=e_{11}, d_{12}\left(e_{1}\right)=0, d_{12}\left(e_{2}\right)=e_{12}, \\
d_{13}\left(e_{0}\right)=e_{12}, d_{13}\left(e_{1}\right)=e_{12} .\end{array}$ & 14 & 103 \\
\hline
\end{tabular}


Table 3: Dimension derivation of Algebra 16.

\begin{tabular}{|c|c|c|c|}
\hline Dimension & Equation(dim Der) & dim Der & No.of equations \\
\hline 14 & $\begin{array}{c}d_{1}\left(e_{0}\right)=e_{0}, d_{1}\left(e_{1}\right)=\frac{13}{2} e_{1}, \\
d_{1}\left(e_{i}\right)=i e_{i}, 2 \leqslant i \leqslant 13 \\
d_{2}\left(e_{i}\right)=0,0 \leqslant i \leqslant 13, d_{3}\left(e_{0}\right)=e_{2}, \\
d_{3}\left(e_{1}\right)=0, d_{3}\left(e_{i}\right)=e_{i+1}, 2 \leqslant i \leqslant 12 \\
d_{4}\left(e_{0}\right)=e_{3}, d_{4}\left(e_{1}\right)=0, d_{4}\left(e_{i}\right)=e_{i+2}, 2 \leqslant i \leqslant 11 \\
d_{5}\left(e_{0}\right)=e_{4}, d_{5}\left(e_{1}\right)=0, d_{5}\left(e_{i}\right)=e_{i+3}, 2 \leqslant i \leqslant 10 \\
d_{6}\left(e_{0}\right)=e_{5}, d_{6}\left(e_{1}\right)=0, d_{6}\left(e_{2}\right)=e_{i+4}, 2 \leqslant i \leqslant 9 \\
d_{7}\left(e_{0}\right)=e_{6}, d_{7}\left(e_{1}\right)=0 d_{7}\left(e_{i}\right)=e_{i+5}, 2 \leqslant i \leqslant 8 \\
d_{8}\left(e_{0}\right)=e_{7}, d_{8}\left(e_{1}\right)=0, d_{8}\left(e_{2}\right)=e_{i+6}, 2 \leqslant i \leqslant 7 \\
d_{9}\left(e_{0}\right)=e_{8}, d_{9}\left(e_{1}\right)=0, d_{9}\left(e_{2}\right)=e_{i+7}, 2 \leqslant i \leqslant 6 \\
d_{10}\left(e_{0}\right)=e_{9}, d_{10}\left(e_{1}\right)=0, d_{10}\left(e_{2}\right)=e_{i+8}, 2 \leqslant i \leqslant 5 \\
d_{11}\left(e_{0}\right)=e_{10}, d_{11}\left(e_{1}\right)=0, d_{11}\left(e_{i}\right)=e_{i+9}, 2 \leqslant i \leqslant 4 \\
d_{12}\left(e_{0}\right)=e_{11}, d_{12}\left(e_{1}\right)=0, d_{12}\left(e_{2}\right)=e_{i+10}, 2 \leqslant i \leqslant 3 \\
d_{13}\left(e_{0}\right)=e_{12}, d_{13}\left(e_{1}\right)=0, d_{13}\left(e_{i}\right)=e_{13}, \\
d_{14}\left(e_{0}\right)=e_{13}, d_{14}\left(e_{1}\right)=e_{13} .\end{array}$ & 15 & 118 \\
\hline 15 & $\begin{array}{c}d_{1}\left(e_{0}\right)=e_{0}, d_{1}\left(e_{1}\right)=7 e_{1}, \\
d_{1}\left(e_{i}\right)=i e_{i}, 2 \leqslant i \leqslant 14 \\
d_{2}\left(e_{i}\right)=0,0 \leqslant i \leqslant 14, d_{3}\left(e_{0}\right)=e_{2}, \\
d_{3}\left(e_{1}\right)=0, d_{3}\left(e_{i}\right)=e_{i+1}, 2 \leqslant i \leqslant 13 \\
d_{4}\left(e_{0}\right)=e_{3}, d_{4}\left(e_{1}\right)=0, d_{4}\left(e_{i}\right)=e_{i+2}, 2 \leqslant i \leqslant 12 \\
d_{5}\left(e_{0}\right)=e_{4}, d_{5}\left(e_{1}\right)=0, d_{5}\left(e_{i}\right)=e_{i+3}, 2 \leqslant i \leqslant 11 \\
d_{6}\left(e_{0}\right)=e_{5}, d_{6}\left(e_{1}\right)=0, d_{6}\left(e_{2}\right)=e_{i+4}, 2 \leqslant i \leqslant 10 \\
d_{7}\left(e_{0}\right)=e_{6}, d_{7}\left(e_{1}\right)=0 d_{7}\left(e_{i}\right)=e_{i+5}, 2 \leqslant i \leqslant 9 \\
d_{8}\left(e_{0}\right)=e_{7}, d_{8}\left(e_{1}\right)=0, d_{8}\left(e_{2}\right)=e_{i+6}, 2 \leqslant i \leqslant 8 \\
d_{9}\left(e_{0}\right)=e_{8}, d_{9}\left(e_{1}\right)=0, d_{9}\left(e_{2}\right)=e_{i+7}, 2 \leqslant i \leqslant 7 \\
d_{10}\left(e_{0}\right)=e_{9}, d_{10}\left(e_{1}\right)=0, d_{10}\left(e_{2}\right)=e_{i+8}, 2 \leqslant i \leqslant 6 \\
d_{11}\left(e_{0}\right)=e_{10}, d_{11}\left(e_{1}\right)=0, d_{11}\left(e_{i}\right)=e_{i+9}, 2 \leqslant i \leqslant 5 \\
d_{12}\left(e_{0}\right)=e_{11}, d_{12}\left(e_{1}\right)=0, d_{12}\left(e_{2}\right)=e_{i+10}, 2 \leqslant i \leqslant 4 \\
d_{13}\left(e_{0}\right)=e_{12}, d_{13}\left(e_{1}\right)=0, d_{13}\left(e_{i}\right)=e_{i+11}, 2 \leqslant i \leqslant 3 \\
d_{14}\left(e_{0}\right)=e_{13}, d_{14}\left(e_{1}\right)=0, d_{14}\left(e_{2}\right)=e_{14}, \\
d_{15}\left(e_{0}\right)=e_{14}, d_{16}\left(e_{1}\right)=e_{14} .\end{array}$ & 16 & 134 \\
\hline 16 & $\begin{array}{c}d_{1}\left(e_{0}\right)=e_{0}, d_{1}\left(e_{1}\right)=\frac{15}{2} e_{1}, \\
d_{1}\left(e_{i}\right)=i e_{i}, 2 \leqslant i \leqslant 15 \\
d_{2}\left(e_{i}\right)=0,0 \leqslant i \leqslant 15, d_{3}\left(e_{0}\right)=e_{2}, \\
d_{3}\left(e_{1}\right)=0, d_{3}\left(e_{i}\right)=e_{i+1}, 2 \leqslant i \leqslant 14 \\
d_{4}\left(e_{0}\right)=e_{3}, d_{4}\left(e_{1}\right)=0, d_{4}\left(e_{i}\right)=e_{i+2}, 2 \leqslant i \leqslant 13 \\
d_{5}\left(e_{0}\right)=e_{4}, d_{5}\left(e_{1}\right)=0, d_{5}\left(e_{i}\right)=e_{i+3}, 2 \leqslant i \leqslant 12 \\
d_{6}\left(e_{0}\right)=e_{5}, d_{6}\left(e_{1}\right)=0, d_{6}\left(e_{2}\right)=e_{i+4}, 2 \leqslant i \leqslant 11 \\
d_{7}\left(e_{0}\right)=e_{6}, d_{7}\left(e_{1}\right)=0 d_{7}\left(e_{i}\right)=e_{i+5}, 2 \leqslant i \leqslant 10 \\
d_{8}\left(e_{0}\right)=e_{7}, d_{8}\left(e_{1}\right)=0, d_{8}\left(e_{2}\right)=e_{i+6}, 2 \leqslant i \leqslant 9 \\
d_{9}\left(e_{0}\right)=e_{8}, d_{9}\left(e_{1}\right)=0, d_{9}\left(e_{2}\right)=e_{i+7}, 2 \leqslant i \leqslant 8 \\
d_{10}\left(e_{0}\right)=e_{9}, d_{10}\left(e_{1}\right)=0, d_{10}\left(e_{2}\right)=e_{i+8}, 2 \leqslant i \leqslant 7 \\
d_{11}\left(e_{0}\right)=e_{10}, d_{11}\left(e_{1}\right)=0, d_{11}\left(e_{i}\right)=e_{i+9}, 2 \leqslant i \leqslant 6 \\
d_{12}\left(e_{0}\right)=e_{11}, d_{12}\left(e_{1}\right)=0, d_{12}\left(e_{2}\right)=e_{i+10}, 2 \leqslant i \leqslant 5 \\
d_{13}\left(e_{0}\right)=e_{12}, d_{13}\left(e_{1}\right)=0, d_{13}\left(e_{i}\right)=e_{i+11}, 2 \leqslant i \leqslant 4 \\
d_{14}\left(e_{0}\right)=e_{13}, d_{14}\left(e_{1}\right)=0, d_{14}\left(e_{2}\right)=e_{i+12}, 2 \leqslant i \leqslant 3 \\
d_{15}\left(e_{0}\right)=e_{14}, d_{15}\left(e_{1}\right)=0, d_{15}\left(e_{2}\right)=e_{14}, \\
d_{16}\left(e_{0}\right)=e_{15}, d_{16}\left(e_{1}\right)=e_{15} .\end{array}$ & 17 & 151 \\
\hline
\end{tabular}




\section{References}

[1] Albeverio, S., Omirov, B. A., Rakhimov, I. S., (2006), Classification of 4-dimensional nilpotent complex Leibniz algebras, Extracta Math., 3(2006), 197-210.

[2] Dixmier. J. and Lister. W. G. , Derivations of nilpotent Lie algebras, Proc. Amer. Math. Soc. 8(1957), 155-158.

[3] M.GOZE AND KHAKIMDJANOV, Nilpotent Lie algebras, printed in the netherlands, (1996), $336 \mathrm{p}$.

[4] Jacobson. N., A note on automorphisms and derivations of Lie algebras, Proc. Amer. Math. Soc. 6(1955), 281-283.

[5] AlnAshri. A. A., Derivations of one type of algebra of First class Filiform Leibniz algebras of Dimension Derivation $(n+1)$, International Journal of Advanced Scientific and Technical ReInternational Journal of Advanced Scientific and Technical Re-
search,Vol.1,No.5,(2015), 41-55.

[6] Loday.J. -L. , Une version non commutative dés algébras de Lie: les algébras de Leibniz, L'Ens. Math., 39 (1993), 269-293.

[7] OMIRov.B. A., On the Derivations of Filiform Leibniz Algebras, Mathematical Notes, 5(2005), 677-685.

[8] Albeverio, S.; Ayupov, Sh. A.; Omirov, B. A., On nilpotent and simple Leibniz algebras, Comm. in Algebra Vol.33(2005), 159-172.

[9] Ayupov, Sh. A.; Omirov, B. A., On Leibniz algebra, Algebra and Operator Theory. Proceeding of the Colloquium in Tashkent (1997), Kluwer (1998), Doi 10.1007/978-94-011-5072-9-1 Springer, p 1-13.

[10] Ayupov, Sh. A.; Omirov, B. A., On 3-dimensional Leibniz algebra, Uzbek Math. (1999),9-14.

[11] AL-hossain, A. A.; Khiyar, A. A.,Derivations of some Filiform Leibniz algebras. pure and Applied mathematics Journal.Vol.3,No.6,(2014),121-125.

[12] Alnashri. A. A., Derivations of Second type of algebra of first class Filiform Leibniz algebras of Dimension Derivation $(n+1)$, International Journal of Advanced Scientific and Technical Research,Vol.3,No.5,(2015), 29-43. 